\title{
Multi-Source Multi-Sensor Image Fusion Based on Bootstrap Approach and SEM Algorithm
}

\author{
Tijani Delleji ${ }^{*}$, Mourad Zribi $^{2}$ and Ahmed Ben Hamida ${ }^{1}$ \\ ${ }^{1}$ Laboratoire d'Electronique et des Technologies de l'Information, Ecole Nationale d'Ingénieurs de Sfax, Tunisia \\ ${ }^{2}$ Laboratoire d'Analyse des Systèmes du Littoral (LASL-EA 2600), Université du Littoral Côte d'Opale, France
}

\begin{abstract}
Bootstrap approach and Stochastic EM algorithm combination applied for the improvement of the multisource and multi-sensor image fusion process; was presented in this research. Improvement concerned not only image quality and reducing processing execution time as mentioned in our previous Bootstrap EM algorithm (BEM), but also regarding initialization dependence as well as fixed classes' number. Such interesting fusion algorithm for multisource and multisensor image using one stochastic phase, i.e. SEM algorithm, preceded by Bootstrap procedure was successfully implemented and tested for several prototype images. Targeted images were firstly split by an unsupervised Bayesian segmentation approach in order to determine a joint region map for the fused image. The Bootstrap approach was then applied to the targeted multisource image in conjunction with the SEM algorithm, forming hence one Bootstrap SEM algorithm called BSEM. The procedure of such algorithm involved both statistical parameters' estimation from one representative Bootstrap sample of each source or sensor images.
\end{abstract}

Keywords: Multi-source multi-sensor image fusion, unsupervised bayesian segmentation, bootstrap approach, sem algorithm.

\section{INTRODUCTION}

The image fusion is of greater importance during the past few years for various applications especially in remote sensing, medical imaging, microscopic imaging, computer vision, and military applications [1]. The objective of multisource and multi-sensor image fusion is to collect observations from various similar or dissimilar sources and sensors, extract the required information of image (inferences) and combine/fuse these with a view to obtain an enhanced status and an identified of a perceived object. So, it is a well known technique by which a set of input images coming from different sensors would be combined together to form one single composite image [2-4]. The fused image contains greater data content for the scene than any one of the individual image sources alone. The source images will usually be collected from different types of sensors, for example, visual cameras, infrared cameras (IR), synthetic aperture radar (SAR), High resolution RADARSAT and panchromatic sensor.

The images generated from these sensors have different characteristics, provide different and complementary information. Thus the procedure of image fusion can enable anyone to perceive features that are impossible to perceive them with any individual type of sensor, thus improving human visual perception. Specialists, who analyze images, use multi-detector manners, to fuse several sets of data, in order to extract the best possible information of a target or a region [5].

\footnotetext{
*Address correspondence to this author at the Electronic Laboratory of Technology's Information (E.L.T.I), National Engineering School of Sfax, BP W 3038 Sfax, Tunisia; Tel: +216 97331 189; Fax: +216 74275 595; E-mail: tijani_delleji@yahoo.fr
}

In recent years, image fusion approaches and their applications have been widely investigated [6-9]. Some researchers focused their attention on studying probabilistic based image fused algorithm. Sharma $[10,11]$ proposed a Bayesian fusion method based on estimation theory and assumes all distortions follow a Gaussian density. Yang [12] presented an algorithm based on the assumption that all distortions best fit Gaussian and non-Gaussian. Lui [3] developed the EM algorithm $[13,14]$ only to estimate fused image in the lowest frequency band. The major problem of these cited methods concerned the "computing time duration", especially for satellite image dotted with a big memory size.

Our previous research [15] concerning Bootstrap EM (BEM) algorithm was one solution for resolving this mentioned problem.

The only disadvantages of this BEM algorithm were the initialization dependence and the fixed classes' number.

This proposed research aimed to give an efficient solution for these above disadvantages met with BEM algorithm. In fact, we tried to combine the Bootstrap approach [16-18] with the Stochastic EM (SEM) algorithm [19, 20].

Bootstrap approach is a re-sampling method that improves estimator properties notably in small samples. Objective of stochastic inserted phase in the algorithm, permitted mainly to determine finite mixture components of probability density functions (pdf).

The presented paper organization could be summarized in the following way. Section II describes the Bootstrap sampling method whereas section III discusses the interest of such approach in image analysis. Bootstrap SEM (BSEM) algorithm for image segmentation and fusion would be presented in section IV. A brief presentation of wavelet decomposition fusion is given in section $\mathrm{V}$. The metrics of quality 
comparison (blind metrics) between the classical EM algorithm and the Bootstrap SEM (BSEM) algorithm are given in section VI. Results of multisource image fusion are shown in section VII followed by a final conclusion.

\section{BOOTSTRAP SAMPLING REVIEW}

Efron [21] introduced the Bootstrap approach as an estimating tool of the statistics sample distribution when standard methods cannot be applied. This approach aim mainly to produce one random sample referred to as Bootstrap sample replacing the original data. The Bootstrap sample is then used to estimate for the targeted parameters, and this procedure (extraction of the random sample and computation of the estimation parameters) is repeated many times in order to create an empirical distribution of the statistic. Such a distribution usually represents a good approximation of the true (and unknown) probability distribution underlying that statistic [18].

Giving for example one sample $X=\left(X_{1}, \ldots \ldots, X_{n}\right)$ having a size $n$, with unknown probability distribution $F$, one could estimate a parameter $\theta$ of $F$ as the mean, the variance...

For doing so, we could generate from $X$ one Bootstrap random sample referred to as $X^{*}=\left(X_{1}^{*}, X_{2}^{*}, \ldots \ldots, X_{n}^{*}\right)$. The simulation could be made according to the empirical distribution function $F_{n}$ of $X$ that gives the probability $1 / n$ for every element. The precision of the estimator could be determined by a simulation of $B$ Bootstrap samples of the considered sample $X$ [22].

\section{BOOTSTRAP FOR IMAGE ANALYSIS}

\section{Principle}

We consider the image as a finite population with $N$ observations referred to as $X=\left(X_{1}, \ldots \ldots, X_{N}\right)$ with $N=N_{l} \times N_{c}$, where $N_{l}$ and $N_{c}$ represent respectively the line number and the columns number of image. Each observation takes its values in a finite set of gray levels $G$. We suppose that the image is our initial sample in its unknown law distribution. From this initial sample, we could apply Bootstrap approach in order to obtain one randomly and with replacement a representative Bootstrap sample referred to as $X^{*}=\left(X_{1}^{*}, \ldots \ldots, X_{n}^{*}\right)$. This model of sampling gives the possibility to simulate a realization that presents a big interest for the evaluation of parameters. Every image would be characterized by a set of different gray levels $G=\left(g_{1}, g_{2}, \ldots . ., g_{K}\right)$, and each gray level $g_{i}$ would characterized by a prior apparition probability $p_{i}$ within the image. This probability could be estimated by its proportion in a Bootstrap sample of optimum size that could be determined by a representative criterion.

\section{Representative Criterion}

Let's consider $N_{1}, N_{2}, \ldots, N_{G}$, as the initial distribution of pixels around gray levels. After bootstrap approach, this initial gray levels' distribution would be then $n_{1}, n_{2}, \ldots, n_{G}$.
Such gray levels distribution could be illustrated as follow Fig. (1).



Fig. (1). The process of sampling.

The numbers $N_{1}, N_{2}, \ldots, N_{G}$ and $n_{1}, n_{2}, \ldots, n_{G}$ verify respectively the following equations:

$$
\begin{aligned}
& N=\sum_{g=1}^{G} N_{g} \\
& n=\sum_{g=1}^{G} n_{g}
\end{aligned}
$$

In our previous work [15], one hybrid criterion was proposed as a process of sampling where the population was distributed in homogeneous sub-groups or in strata $(G)$ and where the drawing of samples was independent in every stratum.

The sub-sample will be called "representative" if it assures the proportional distribution of individuals, that is to say:

$\frac{n_{g}}{n}=\frac{N_{g}}{N} \Rightarrow \tau_{g}=\frac{n_{g}}{N_{g}}=\frac{n}{N} \Rightarrow n=N \times \tau_{g}$

Improving more the expression of $n$ :

$G \times n=N \times \sum_{g=1}^{G} \frac{n_{g}}{N_{g}} \Rightarrow n=\frac{N}{G} \times \sum_{g=1}^{G} \frac{n_{g}}{N_{g}}$

After that, we could apply one of the criterions given in [18] to determine the optimal size of a sub-sample.

In this work, we choose the following criterion: An observed sample $\left(x_{1}^{*}, x_{2}^{*}, \ldots ., x_{n}^{*}\right)$ of $n$ size would be representative of the entire image only if each gray level appears at least one time during $n$ drawing, thus the following expression is deduced:

$n_{g}>4 \times K_{g}$

$K_{g}$ represents the gray level number in a $g$ stratum.

The expression of $n$ will be in the following form:

$$
n>\frac{4 N}{G} \times \sum_{g=1}^{G} \frac{K_{g}}{N_{g}}
$$

\section{BSEM ALGORITHM APPLICATION}

Many researchers have applied the Bootstrap approach in pattern classification $[16,18,23]$ and in image segmentation [15]. In this section, we present the application of BSEM algorithm firstly for unsupervised image segmentation, and secondly for image fusion process. 


\section{Unsupervised Image Segmentation by BSEM Algorithm}

Image segmentation is an important early vision task where pixels with similar features are grouped into homogeneous regions (classes). The basis of any statistical pattern classification system is a known parametric or nonparametric probability density of the feature vector of each class [24]. For images, feature vectors $\mathrm{X}$ are the gray levels of the pixels. The unsupervised Bayesian image segmentation [24, 25] consists of the following two important steps: The first one is the mixture identification. It can be achieved either by the expectation-maximization (EM) $[26,27]$ or by the Stochastic EM (SEM) [19, 20]. The second step consists of the application of the Bayesian rule. Here, we propose to use SEM algorithm and Bootstrap approach in unsupervised Bayesian segmentation.

The BSEM algorithm is proposed to estimate the parameters of mixture from a representative Bootstrap sample of a given image [15]. The mixture density of a random variable $X^{*}=\left(X_{1}^{*}, \ldots \ldots, X_{n}^{*}\right)$ with an observed result $x^{*}=\left(x_{1}^{*}, x_{2}^{*}, \ldots . ., x_{n}^{*}\right)$ is defined by the following form:

$g\left(x^{*}\right)=\sum_{k=1}^{K} \pi_{k} f\left(x^{*} / \theta_{k}\right)$

with: $0 \leq \pi_{k} \leq 1$, and $\sum_{k=1}^{K} \pi_{k}=1$,

where $\mathrm{K}$ the number of classes, $f\left(x^{*} / \theta_{k}\right)$ the Gaussian density, and $\theta_{k}=\left(\mu_{k}, \Sigma_{k}\right)^{t}$ the parameters of mixture.

The application of the BSEM algorithm to image segmentation consists of the following phases:

a) Determination of the optimal size of Bootstrap sample.

b) Drawing with replacement of a representative Bootstrap sample, with a size $n<N$ from the totality of the image.

c) Resampling of $B$ samples from the initial representative sample.

d) Application of the SEM algorithm to estimate parameters of the simulated Bootstrap samples.

e) Classification: The Bayesian Rule (BR).

After the mixture identification, the BR is applied in order to classify the pixels according to their gray level $x^{*}$ :

$k\left(x^{*}\right)=\arg \left[\max _{1 \leq k \leq K}\left\{\pi_{k} f\left(x^{*} / \theta_{k}\right)\right\}\right]$

where $k\left(x^{*}\right)$ represents the class label of the pixel $x^{*}$.

Regarding phase d), we could notice the following steps for parameters' estimation [28]:

\section{(i) Initialization}

The first step is the initialization of a priori probabilities, averages and variances of every class. With the help of the image histogram or the K-Means Clustering method, we initialize those parameters in the following way: $\hat{\pi}_{k}^{0}=\frac{\hat{n}_{k}^{0}}{n} ; \hat{\theta}_{k}^{0}=\left(\mu_{k}^{0}, \Sigma_{k}^{0}\right)^{t}$

where $\hat{n}_{k}^{0}$ is the total number of observations in the class $k$.

\section{(ii) E Step}

In this step we estimate the a posterior distribution $\hat{p}_{k}^{m}\left(x_{i}^{*}\right)$ for the pixel $x_{i}^{*}$ that belongs to the class $k$ at the $m^{\text {th }}$ iteration by:

$\hat{p}_{j}^{m}\left(x_{i}\right)=\frac{\hat{\pi}_{j}^{m} f\left(x_{i} / \hat{\theta}_{j}^{m}\right)}{\sum_{l=1}^{K} \hat{\pi}_{l}^{m} f\left(x_{i} / \hat{\theta}_{l}^{m}\right)}$

where $f\left(x_{i} / \hat{\theta}_{j}^{m}\right)$ designates the Gaussian density corresponding to $\hat{\mu}_{j}^{m}$ and $\hat{\Sigma}_{j}^{m}$.

\section{(iii) S Step}

A partition $\hat{r}^{m}=\left(\hat{r}_{1}^{m}, \ldots \ldots . ., \hat{r}_{K}^{m}\right)$ of $x_{1}^{*}, \ldots \ldots ., x_{n}^{*}$ is designed by assigning each point $x_{i}^{*}$ at random to one of the mixture components according to the multinomial distribution with parameter $\left(\hat{p}_{j}^{m}\left(x_{i}^{*}\right), 1 \leq j \leq K\right)$.

\section{(iv) Maximization}

The SEM algorithm supposes that every $x_{i}^{*}$ belonging to $\hat{r}_{j}^{m}$ for each $j=1, \ldots, K$, is realized according to the $j^{\text {th }}$ mixture component $f\left(x_{i}^{*} / \theta_{j}\right)$. By denoting $\hat{C}_{j}^{m}=\operatorname{card}\left(\hat{r}_{j}^{m}\right)$ (the cardinal of $\left.\hat{r}_{j}^{m}\right), \hat{r}_{j}^{m}=\left(x_{1, j}^{m}, x_{2, j}^{m}, \ldots \ldots \ldots, x_{\hat{C}_{j}^{m}, j}^{m}\right)$, we can estimate the a prior probability $\hat{\pi}_{j}^{m+1}$, the mean $\hat{\mu}_{j}^{m+1}$ and the covariance matrix $\hat{\Sigma}_{j}^{m+1}$ by:

$\hat{\pi}_{j}^{m+1}=\frac{\hat{C}_{j}^{m}}{n}$,

$\hat{\mu}_{j}^{m+1}=\frac{1}{\hat{C}_{j}^{m}} \sum_{i=1}^{\hat{C}_{j}^{m}} x_{i, j}^{m}$

and

$\hat{\Sigma}_{j}^{m+1}=\frac{1}{\hat{C}_{j}^{m}} \sum_{i=1}^{\hat{C}_{j}^{m}}\left(x_{i, j}^{m}-\hat{\mu}_{j}^{m+1}\right)\left(x_{i, j}^{m}-\hat{\mu}_{j}^{m+1}\right)^{t}$

The algorithm stops when the condition:

$\left|\hat{\pi}_{k}^{m+1}-\hat{\pi}_{k}^{m}\right|<\varepsilon$

is satisfied, with $\varepsilon$ is a given small number.

\section{Region Analysis}

The above unsupervised segmentation using the BSEM algorithm is applied separately to each source image to obtain region maps for each of them. The region maps of different sensor images are generally different, because these images can come from diverse modalities. Input images may 
contain different objects which may appear with different shapes on input images [29]. Thus we need to construct a joint region map for the fused image by combining region map I to region map II. For the satellite images, (they are very texture) which were come from different sensors; some undesired small regions may be created in the joint region map. These undesired small regions may not contain enough information and result an artifacts in the fused image. To deal with this problem, we use a merging technique to merge those undesired small regions into the neighboring regions (see Fig. 2).

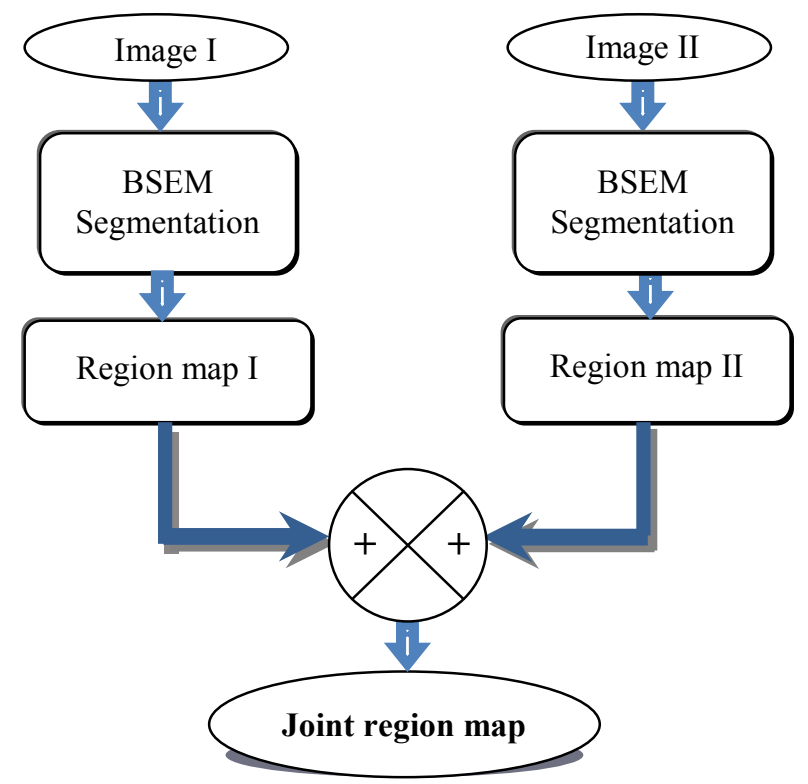

Fig. (2). Conception diagram of the joint region map.

\section{Image Formation Model}

We assume that the input images are modeled as the true scene corrupted by a Gaussian mixture noise. To determine the parameters of distortion, we apply a new method called BSEM fusion algorithm.

BSEM algorithm requires the image formation model which is determined for each region in the joint region map. This model is defined for each pixel $l=1, \ldots \ldots, M$ in a region [3] as:

$z_{i}(l)=\beta_{i} s(l)+\alpha_{i}+\varepsilon_{i}(l)$

where $i=1, \ldots . ., q$ indexes the sensors; $M$ is region size; $z_{i}(l)$ is the input sensor image region; $s(l)$ is the true scene region which we hope to estimate using the fused method. The parameters $\alpha_{i}$ and $\beta_{i}$ will be considered to be the same for each coefficient $(l=1, \ldots, M)$ in the region. $\beta_{i}=+1,-1$ or 0 is the sensor selectivity factor which indicate that the model acknowledges that a given sensor may be able to "see" this object $\left(\beta_{i}=+1\right)$, may fail to "see" this object $\left(\beta_{i}=0\right)$, or may "see" this object with a polarity reversed representation ( $\left.\beta_{i}=-1\right), \alpha_{i}$ is the sensor bias or the formation distortions of sensors and $\varepsilon_{i}(l)$ is the random distortion. The noise is modeled as a $K$-term mixture of Gaussian probability functions (pdfs) as:

$f_{\varepsilon_{i}(l)}\left(\varepsilon_{i}(l)\right)=\sum_{k=1}^{K} \lambda_{k, i}(l) \frac{1}{\sqrt{2 \pi \sigma_{k, i}^{2}(l)}} e^{-\frac{\varepsilon_{i}(l)^{2}}{2 \sigma_{k, i}^{2}}}$ where $\lambda_{k, i}(l)$ and $\sigma_{k, i}(l)$ are the distortion parameters. They will be considered the same for each element in the image region.

\section{BSEM Fusion Procedure}

BSEM algorithm proposes to estimate the model parameters from representative Bootstrap samples of each region of images (see Fig. 3). Therefore, instead of considering the region like an observation sample as the fact of the classic EM algorithm, the BSEM replaces the observed region $z=\left(z_{1}, z_{2}, \ldots \ldots . ., z_{M}\right)$ that has a size equal to $M$ by a $L$-size representative Bootstrap sample $z^{*}=\left(z_{1}^{*}, z_{2}^{*}, \ldots \ldots . ., z_{L}^{*}\right)$ selected randomly.

The application of the BSEM algorithm to images regions consists in the following phases:

a) Determination of the optimal size of Bootstrap sample of a region.

b) Drawing with replacement of a representative Bootstrap sample $\mathrm{z}_{\mathrm{i}}^{*}(1)$ of size $L<M$ from the totality of a input images regions $\mathrm{z}_{\mathrm{i}}(\mathrm{l})$.

c) Resampling of $B$ samples from the initial representative sample $\mathrm{z}_{\mathrm{i}}{ }^{*}(1)$.

d) Application of the SEM algorithm to estimate parameters of the simulated bootstrap samples.

e) Using the complete regions of input images, recalculate $g_{k, i, l}\left(z_{i}(l)\right)$ and the true region $s(l)$ with $l=1, \ldots ., M$.

f) Assembled the updated regions to obtain the fused image.

Fig. (3) explains the sequence of phases of the BSEM algorithm.

Regarding phase d), we could notice the following steps $[12,29]$ :

\section{Initialization of Parameters}

The initial values of the parameters are needed to start the SEM algorithm. We choose the initial estimations for the sample of true scene region that comes from the weighted average of the Bootstrap samples of input images regions.

$$
s^{*}(l)=\sum_{i=1}^{q} w_{i} \cdot z_{i}^{*}(l) ; l=1, \ldots ., L
$$

where $\sum_{i=1}^{q} w_{i}=1$.

The parameter $w_{i}$ is the weight for each Bootstrap sample image region. A simple initialization of $\beta_{i}$ is to assume that the region appears in each sensor image $[3,12]$. The initialization for the sensor bias $\alpha_{i}$ is to come from the average of gray level of each source image-region.

2. Estimation of the conditional distribution $g_{k, i, l}\left(z_{i}^{*}(l)\right)$ by:

$g_{k, i, l}\left(z_{i}^{*}(l)\right)=\frac{\lambda_{k, i} f_{k}\left(z_{i}^{*}(l)\right)}{\sum_{k=1}^{K} \lambda_{k, i} f_{k}\left(z_{i}^{*}(l)\right)}$ 
where,

$$
f_{k}\left(z_{i}^{*}(l)\right)=\frac{e^{-\frac{\left(z_{i}^{*}(l)-\beta_{i} s^{*}(l)-\alpha_{i}\right)}{2 \sigma_{k, i}^{2}}}}{\sqrt{2 \pi \sigma_{k, i}^{2}}}
$$

\section{Stochastic Step}

In this step, we construct a random variable of Bernoulli introducing the random disturbances that are going to make the BSEM fusion algorithm converge quickly toward the good solution.

The variable of Bernoulli is defined by:

$$
\left\{\begin{aligned}
u_{k, i, l}= & 1 \text { if the term } k \text { is drawn according to the multinomial } \\
& \quad \text { law with parameter, } g_{k, i, l}\left(z_{i}^{*}(l)\right), 1 \leq k \leq K \\
u_{k, i, l}= & 0 \text { if not }
\end{aligned}\right.
$$

\section{Update Selectivity Factors: $\beta_{i}$ for $i=1, \ldots, q$.}

The parameter $\beta_{i}$ is chosen to have the value from the set $\{-1,0,1\}$ that maximizes

$$
\begin{aligned}
& Q=-\frac{1}{2} \sum_{i=1}^{q} \sum_{l=1}^{L} \sum_{k=1}^{K}\left(\ln \left(\sigma_{k, i}^{2}\right)+\frac{\left(z_{i}^{*}(l)-\beta_{i} s^{*}(l)-\alpha_{i}\right)^{2}}{\sigma_{k, i}^{2}}\right) . u_{k, i, l} \\
& \mathrm{i}=1, \ldots \ldots, \mathrm{q} ; \mathrm{k}=1, \ldots . ., \mathrm{K} ; \mathrm{l}=1, \ldots . ., \mathrm{L}
\end{aligned}
$$

5. Update the Bootstrap Sample Region s* (l) of a Scene

$$
s^{*}(l)=\frac{\sum_{i=1}^{q} \sum_{k=1}^{K}\left(\left(z_{i}^{*}-\alpha_{i}\right) \beta_{i} \frac{u_{k, i, l}}{\sigma_{k, i}^{2}}\right)}{\sum_{i=1}^{q} \sum_{k=1}^{K} \beta_{i}^{2} \frac{u_{k, i, l}}{\sigma_{k, i}^{2}}}
$$

6. Update the Distortions Parameters: $\lambda_{k, i}, \sigma_{k, i}^{2}$ and $\alpha_{i}$.

$\lambda_{k, i}=\frac{1}{L} \sum_{l=1}^{L} u_{k, i, l}$

$\sigma_{k, i}^{2}=\frac{\sum_{l=1}^{L}\left(z_{i}^{*}(l)-\beta_{i} s^{*}(l)-\alpha_{i}\right)^{2} u_{k, i, l}}{\sum_{l=1}^{L} u_{k, i, l}}$

$\alpha_{i}=\frac{\sum_{l=1}^{L} \sum_{k=1}^{K}\left(z_{i}^{*}(l)-\beta_{i} s^{*}(l)\right) \frac{u_{k, i, l}}{\sigma_{k, i}^{2}}}{\sum_{l=1}^{L} \sum_{k=1}^{K} \frac{u_{k, i, l}}{\sigma_{k, i}^{2}}}$

\section{Repeat Steps 2 to 5}

Using the new values of parameters: $\mathrm{s}^{*}(1), \beta_{i}, \lambda_{k, i}, \sigma_{k, i}^{2}$ and $\alpha_{i}$.

\section{WAVELET DECOMPOSITION FUSION}

Wavelet transform (WT) is a mathematical tool developed in the field of signal processing. Wavelet decomposition based image fusion involves three steps; forward transform coefficient combination and backward transform. In the forward transform, two or more registered input images are calculated to get their wavelet coefficients. These coefficients respectively represent the approximation, horizontal, vertical and diagonal components of the input images [30].

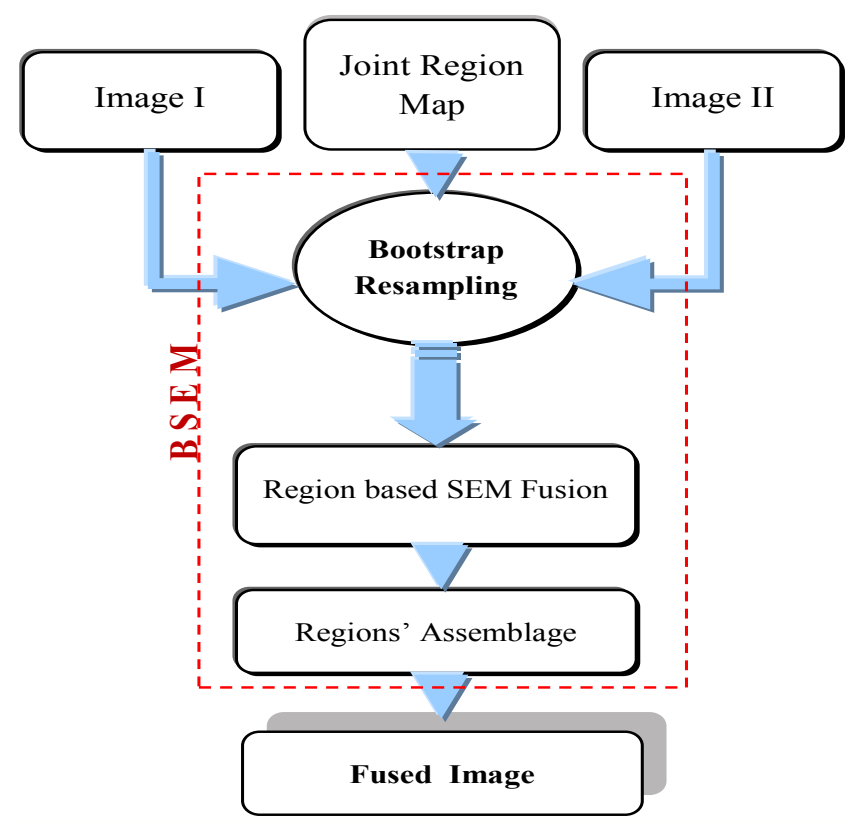

Fig. (3). BSEM fusion procedure diagram.

Then, these wavelet coefficients from the different input images are combined according to certain fusion rules to get fused wavelet coefficients, after that a wavelet transform reconstruction (WTR) is applied to obtain finally a fused image (see Fig. 4).

We notice that the images fused by most wavelet-based methods have less quality because the critical down sampling is included in the wavelet transform. It is reasonable that the BSEM and other methods [31] of fusion are better than all other including WT and SEM.

\section{PERFORMANCE EVALUATION}

The widespread use of image analysis (segmentation, fusion, etc) methods, in military applications, in medical diagnostics, in remote sensing, etc, has led to a rising demand of applicable quality of evaluation metrics in order to compare the results gotten with different algorithms [32]. Quality evaluation of images processing is often carried out by human visual inspection [33].

In this work, we applied the objective fusion performance measures (blind metrics) which were introduced by Pielle and Cvejic [27, 32]. Piella and Cvejic used the image quality index $Q_{0}$ that was introduced by Wang and Bovik in [34] to define some new objective measures for image fusion which do not require a reference image.

\section{The Fusion Quality Index $Q(a, b, f)$}

Piella [32] denote by $Q(a, b, f)$ the quality index, where $a$ and $b$ are the two input images and $f$ is the fused image.

$s(a \backslash w)$ is the some saliency of image $a$ in a window $w$. The $s(a \backslash w)$ should reflect the local relevance of image $a$ within the window $w$, and it may depend on (e.g. contrast, sharpness, or entropy). We note by $\lambda(w)$ the local weight that indicates the relative importance of image $a$ compared to image $b$. A typical choice for $\lambda(w)$ is: 


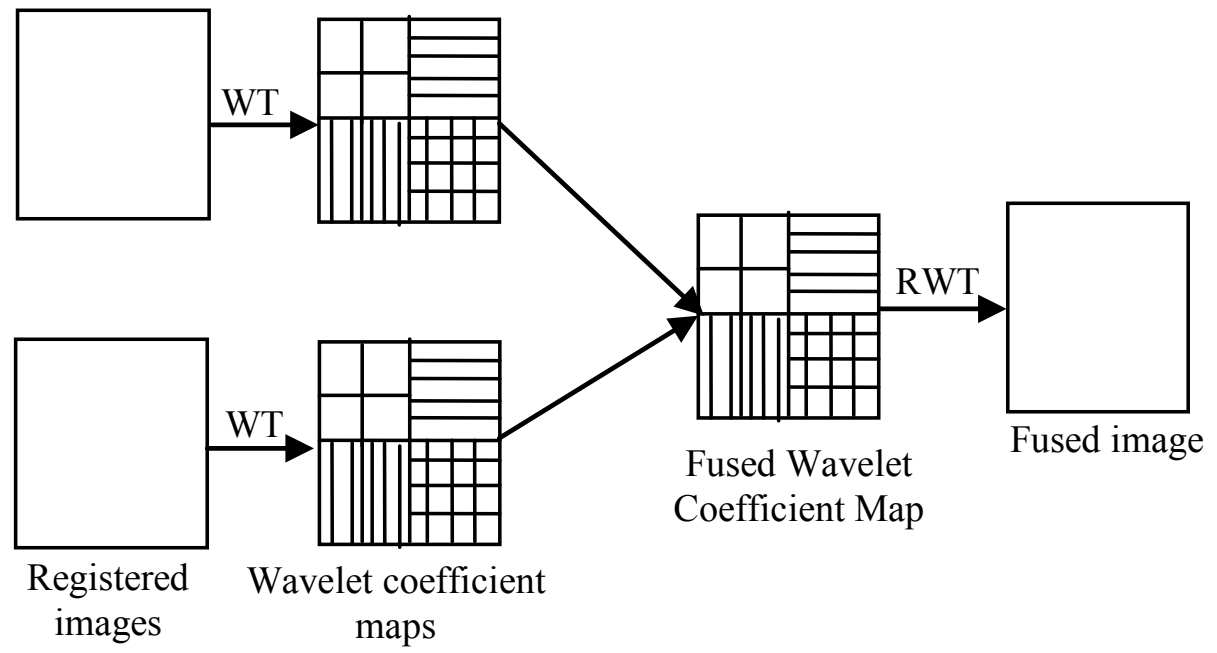

Fig. (4). The block diagram of the image fusion scheme.

$\lambda(w)=\frac{s(a \backslash \mathrm{w})}{s(a \backslash \mathrm{w})+s(b \backslash \mathrm{w})}$

Then, $Q(a, b, f)$ is defined as:

$$
\begin{aligned}
& Q(a, b, f)= \\
& \quad \frac{1}{|W|} \sum_{w \in W}\left(\lambda(w) Q_{0}(a, f \mid w)+(1-\lambda(w)) Q_{0}(b, f \mid w)\right)
\end{aligned}
$$

where $W$ is the family of all windows.

\section{The Fusion Quality Index $Q w(a, b, f)$}

From Eq. (26), we define the weighted fusion quality index $Q w(a, b, f)$ by:

$Q_{W}(a, b, f)=\sum_{w \in W} c(w)\left(\lambda(w) Q_{0}(a, f \mid w)+(1-\lambda(w)) Q_{0}(b, f \mid w)\right)$

where,

$c(w)=\frac{C(w)}{\sum_{w^{\prime} \in W} C\left(w^{\prime}\right)}$

and,

$C(w)=\max (s(a \backslash w)+\mathrm{s}(\mathrm{b} \backslash w))$

We choose the variance and the entropy to calculate the relevance $s(a \backslash w)$ of an image. The variance denoted by $\sigma_{x}{ }^{2}$ is an arbitrary measurement being used in order to characterize the dispersion (the measure of the homogeneity) of the pixels values of an image (or a window ( $w)$ in our case) that is defined by:

$$
\sigma_{x}^{2}=\frac{1}{n-1} \sum_{i=1}^{n}\left(x_{i}-\bar{X}\right)^{2}
$$

With

$$
\bar{X}=\frac{1}{n} \sum_{i=1}^{n} x_{i}
$$

where $n$ is the image size; $x_{i}$ the pixel value; and $\bar{X}$ the mean of pixels values in a window.
The Entropy denoted by $(\mathrm{H})$ of an image is the measurement of information which is present in an image (or in a window) and is defined as following description [35]:

$$
H=-\sum_{i=0}^{L} p(i) \log _{2} p(i)
$$

where $L$ is the gray level of the image, $p(i)$ is the ratio of the number of pixels $n_{i}$ with gray level $i$ and the total number $n$ of pixels in the image; $p(i)=n_{i} / n$.

\section{The Fusion Quality Index $Q b(a, b, f)$}

Cvejic [36] denotes by $Q b(a, b, f)$ a novel fusion performance measure that takes into account the similarity between the input image block and the fused image block within the same spatial position. It is defined as:

$$
\begin{aligned}
& Q_{b}(a, b, f)= \\
& \left.\quad \sum_{w \in W} \operatorname{sim}(a, b, f \mid w)\left(Q_{0}(a, f \mid w)-Q_{0}(b, f \mid w)\right)+Q_{0}(b, f \mid w)\right)
\end{aligned}
$$

Cvejic [36] define $\operatorname{sim}(a, b, f \mid w)$ as :

$$
\operatorname{sim}(a, b, f \mid w)= \begin{cases}0 & \text { if } \frac{\sigma_{a f}}{\sigma_{a f}+\sigma_{b f}}<0 \\ \frac{\sigma_{a f}}{\sigma_{a f}+\sigma_{b f}} & \text { if } 0 \leq \frac{\sigma_{a f}}{\sigma_{a f}+\sigma_{b f}} \leq 1 \\ 1 & \text { if } \frac{\sigma_{a f}}{\sigma_{a f}+\sigma_{b f}}>1\end{cases}
$$

where

$\sigma_{u v}=\frac{1}{n-1} \sum_{i=1}^{n}\left(u_{i}-\bar{u}\right)\left(v_{i}-\bar{v}\right)$

Each analysis window is weighted by the $\operatorname{sim}(a, b, f \mid w)$ that is dependent on the similarity in spatial domain between the input image and the fused image [36]. 


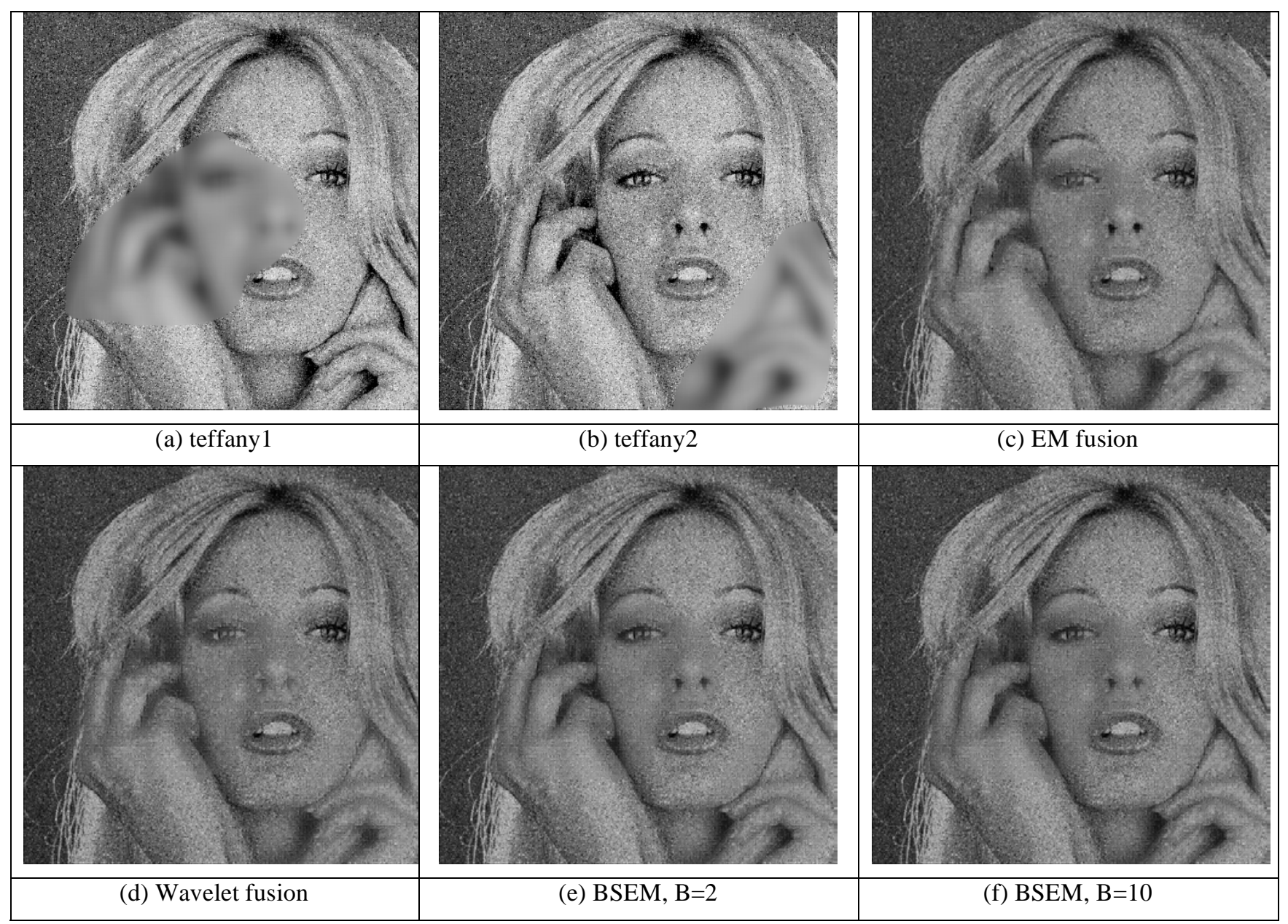

Fig. (5). The fused results of "teffany1" and "teffany2" images.

\section{RESULTS AND DISCUSSION}

To assess the performance of the new approach (BSEM) we consider some pairs of real images. These images are represented by 256 gray levels.

The pairs of test images included:

\section{"Teffany" Pair of Images} tion.

This pair is a multifocus images of $512 \times 512$ pixel resolu-

Input image "teffanyl" is the original image "teffany" with added noise and a blurred segment.

Input image "teffany2" is the original image "teffany" with added noise and another blurred segment.

\section{Night Vision Pair of Images}

This pair of images shows an example of night vision application. These images are $360 \times 270$ pixel resolution. The IR (infrared) Image provides considerable application including showing a person, while the visual image shows the building.

\section{AVIRIS and RADARSAT Pair of Images}

The two input images (AVIRIS image, band 183 and High resolution RADARSAT image) are from two different sensors: AVIRIS and RADARSAT. Although they depict the same region, each image contains complementary information. These images are $400 \times 350$ pixel resolution.

Fig. (5c) shows the fused results of Fig $(\mathbf{5 a}, \mathbf{b})$ images, using classical EM.

Fig. (6c, d) show the segmented images of Fig. (6a, b) respectively. Fig. (6e) shows the joint region map of the segmented images (Fig. 6c, d). Fig. (6f) is the fused image using the classical EM fusion algorithm.

Fig. (7) shows the same description as Fig. (6).

In order to decrease the great dependence of neighbor pixels of the real images, it is more suitable to select randomly representative samples from the regions of input images instead of considering the total number of pixels. After that, we generate some B re-samples (artificial samples) from these representatives' samples. In Figs. $(\mathbf{5}, \mathbf{7})$ we view also the fused images obtained by using the BSEM fusion algorithm; with a variation of the number of artificial samples $B$ ( the choice of $B$ is arbitrary). This kind of sample selection considerably reduces the fusion time (see: Table 4). The sample selection process in BSEM leads to a great improvement in maximum likelihood parameter estimation. From which comes the improvement of the fused image quality (see: Tables 1-3). 

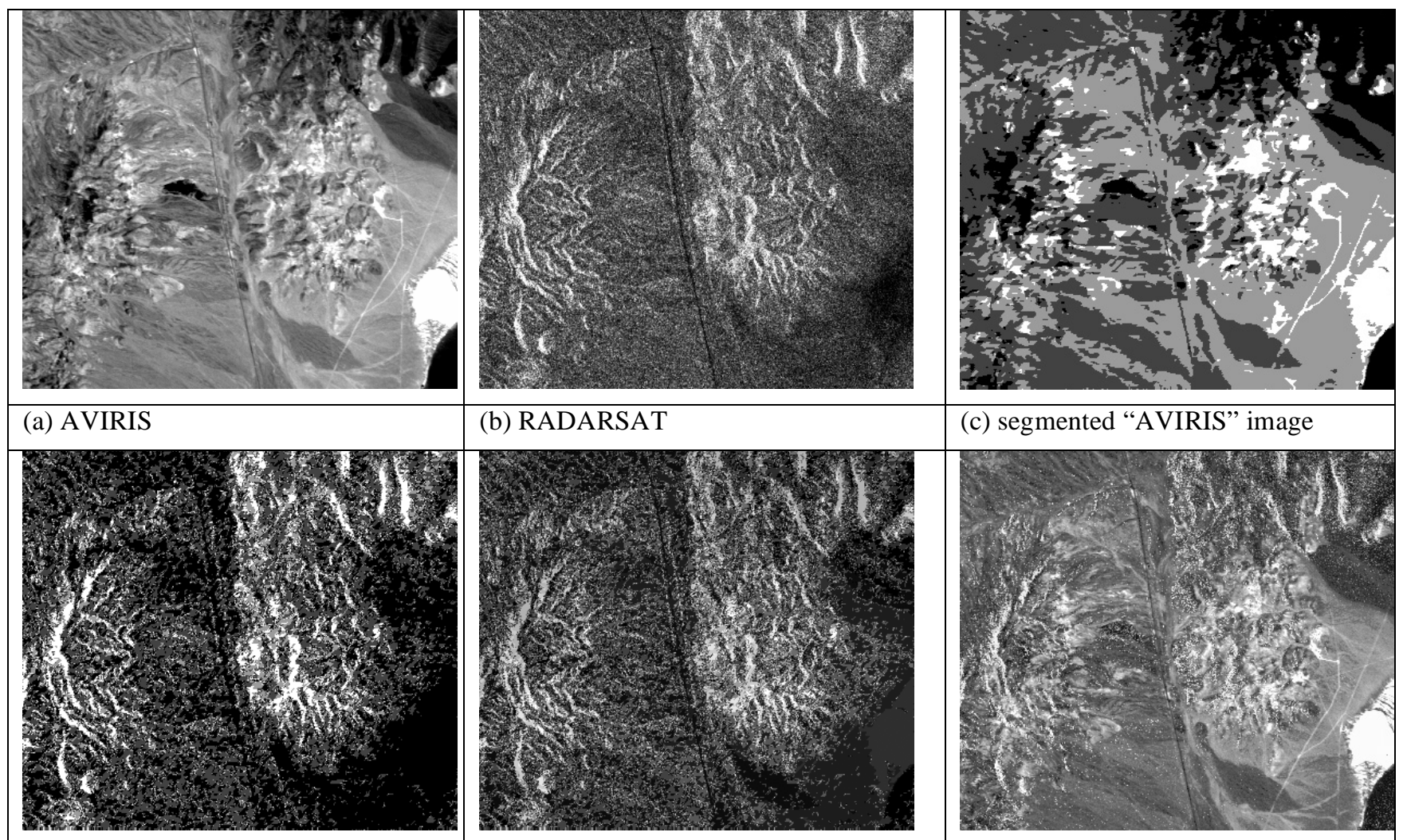

(c) segmented "AVIRIS" image

(d) segmented "RADARSAT" image

(e) joint region map
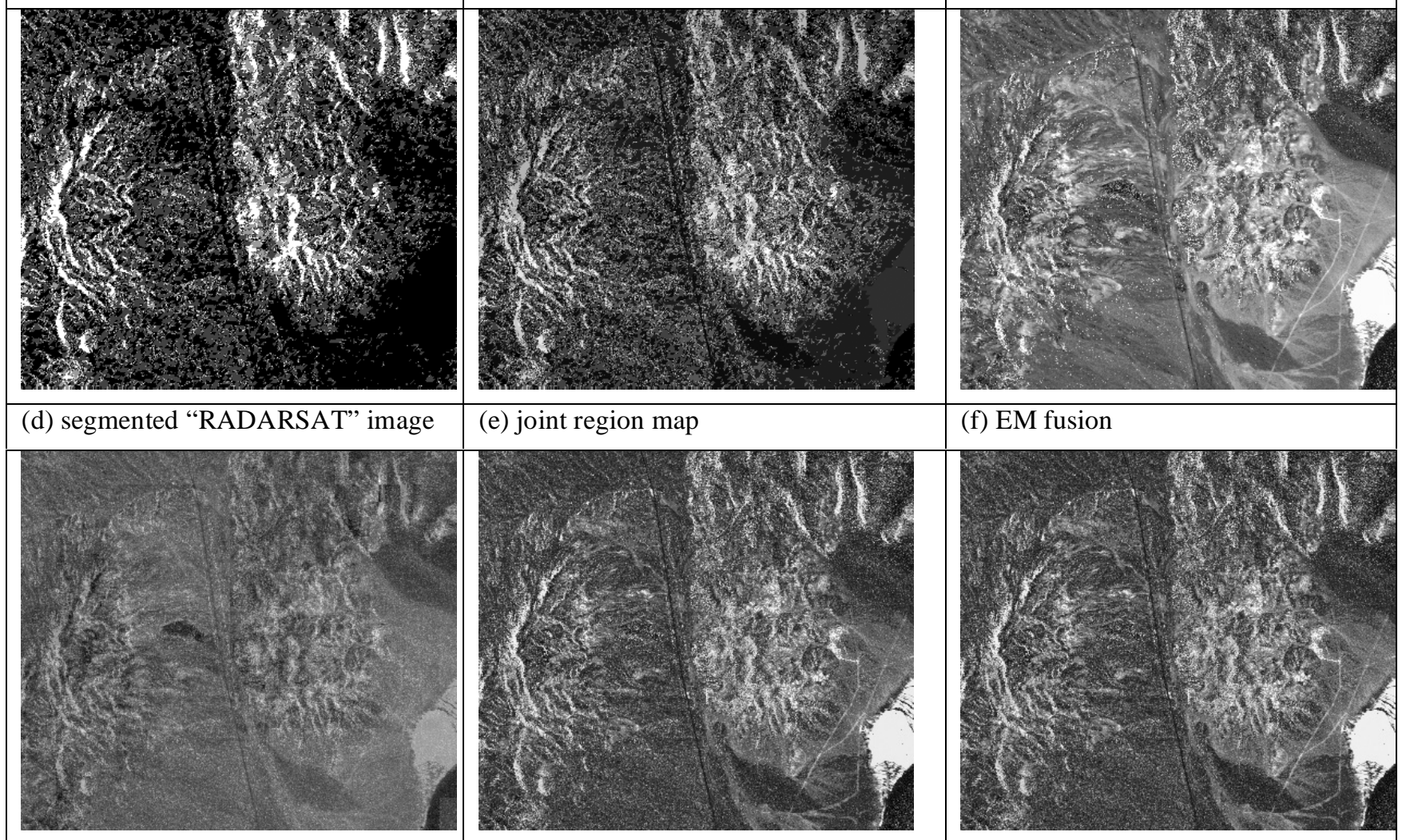

(f) EM fusion

(g) Wavelet fusion

(h) BSEM, B=2

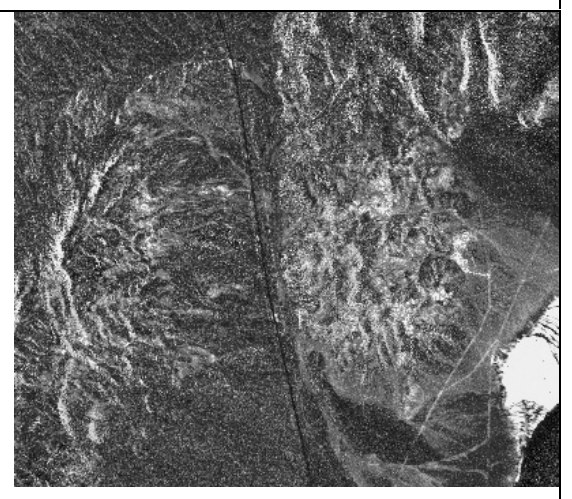

(i) $\mathrm{BSEM}, \mathrm{B}=10$

Fig. (6). The fused results of "AVIRIS" and "RADARSAT" images.

Table 1. Comparison Between Different Quality Measures for the Composite Images in Fig. (5).

\begin{tabular}{|c|c|c|c|c|}
\hline Metrics & $\begin{array}{c}\text { Wavelet } \\
\text { (db2) }\end{array}$ & EM & $\begin{array}{c}\text { BSEM, } \\
\mathbf{B = 2}\end{array}$ & $\begin{array}{c}\text { BSEM, } \\
\mathbf{B = 1 0}\end{array}$ \\
\hline \hline Q_variance & 0.7881 & 0.7859 & 0.7950 & 0.7944 \\
\hline Qw_variance & 0.7978 & 0.7955 & 0.8031 & 0.8025 \\
\hline Q_entropy & 0.8016 & 0.7993 & 0.8088 & 0.8082 \\
\hline Qw_entropy & 0.7980 & 0.7962 & 0.8056 & 0.8050 \\
\hline Qb & 0.8426 & 0.8383 & 0.8566 & 0.8558 \\
\hline
\end{tabular}

The fusion quality indexes: $\mathrm{Q}, \mathrm{Q}_{\mathrm{w}}$ and $\mathrm{Q}_{\mathrm{b}}$ are based on $\mathrm{Q}_{0} \cdot \mathrm{Q}_{0}$ is the image quality index that is used it to quantify the structural distortion between images $\mathbf{a}$ and $\mathbf{b}$. In fact, the value $\mathrm{Q}(\mathrm{a}, \mathrm{b})$ is a measure for the similarity of images a and $\mathrm{b}$; and takes values between -1 and 1 . This quality index models image distortions as a combination of three different factors: loss of correlation, luminance distortion and contrast distortion. These three factors are according to the parameters: the mean and the variance (see eq. 30 and 31). The variance is used to measure the dispersion and the homogeneity in an image. These two characteristics don't follow a linear function in a textured image, as the multi-source or the multi-sensor images. Consequently, $\mathrm{Q}, \mathrm{Q}_{\mathrm{w}}$ and $\mathrm{Q}_{\mathrm{b}}$ are nonlinear functions and that small changes in its values already indicated a large improvement.

In this work, and for better evaluation of our new approach, we also apply the Daubechies wavelet (db2) to fuse prototype images. 


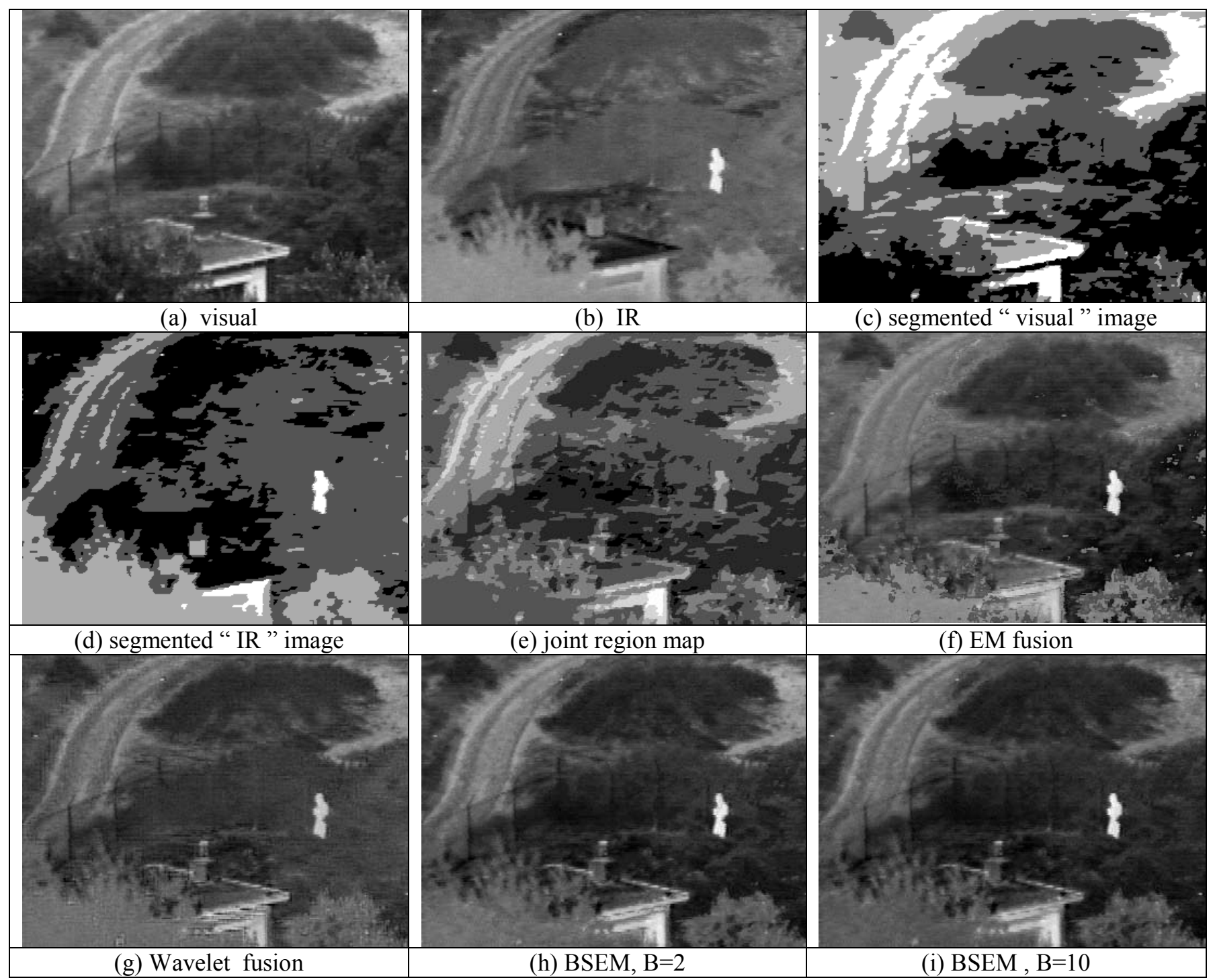

Fig. (7). The fused results of "visual" and "IR" images.

Table 2. Comparison Between Different Quality Measures for the Composite Images in Fig. (6).

\begin{tabular}{|c|c|c|c|c|}
\hline Metrics & Wavelet & EM & $\begin{array}{c}\text { BSEM, } \\
\mathbf{B = 2}\end{array}$ & $\begin{array}{c}\text { BSEM, } \\
\mathbf{B}=10\end{array}$ \\
\hline \hline Q_variance & 0.6458 & 0.5105 & 0.7485 & 0.7267 \\
\hline Qw_variance & 0.6653 & 0.5339 & 0.7818 & 0.7590 \\
\hline Q_entropy & 0.5094 & 0.5424 & 0.5375 & 0.5328 \\
\hline Qw_entropy & 0.5099 & 0.5431 & 0.5384 & 0.5338 \\
\hline Qb & 0.6134 & 0.7000 & 0.7668 & 0.7599 \\
\hline
\end{tabular}

Table 3. Comparison Between Different Quality Measures for the Composite Images in Fig. (7).

\begin{tabular}{|c|c|c|c|c|}
\hline Metrics & Wavelet & EM & $\begin{array}{c}\text { BSEM, } \\
\text { B=2 }\end{array}$ & $\begin{array}{c}\text { BSEM, } \\
\text { B=10 }\end{array}$ \\
\hline \hline Q_variance & 0.7180 & 0.6113 & 0.7139 & 0.7278 \\
\hline Qw_variance & 0.6859 & 0.6638 & 0.7270 & 0.7532 \\
\hline Q_entropy & 0.6398 & 0.5435 & 0.6528 & 0.6644 \\
\hline Qw_entropy & 0.6318 & 0.5358 & 0.6474 & 0.6593 \\
\hline Qb & 0.7720 & 0.7854 & 0.7933 & 0.7871 \\
\hline
\end{tabular}


Table 4. Time of Fusion Image Under a Compute Station

\begin{tabular}{|c|c|c|c|c|}
\hline $\begin{array}{c}\text { Pair of } \\
\text { Images }\end{array}$ & $\begin{array}{c}\text { Pixel } \\
\text { Resolution }\end{array}$ & $\begin{array}{c}\text { Time in } \\
\text { Seconds of } \\
\text { Fusion } \\
\text { Approach } \\
\text { Algorithm } \\
\text { EM }\end{array}$ & $\begin{array}{c}\text { BSEM, } \\
\text { B=2 }\end{array}$ & $\begin{array}{c}\text { BSEM, } \\
\text { B=10 }\end{array}$ \\
\hline \hline Teffany & $512 \times 512$ & 6975.88 & 1181.75 & 2012.24 \\
\hline $\begin{array}{c}\text { AVIRIS and } \\
\text { RADARSAT }\end{array}$ & $400 \times 350$ & 1081.04 & 234.71 & 411.31 \\
\hline Night vision & $360 \times 270$ & 724.67 & 115.09 & 285.75 \\
\hline
\end{tabular}

We use some evaluation performance metrics, we conclude that the BSEM is better than the WT and the EM approaches concerning the enhancement of the fused image.

\section{CONCLUSIONS}

A new image fusion method combining the Bootstrap approach with the stochastic EM algorithm was presented in this paper. We tried to exploit performances of Bootstrap sampling model that has been used especially in the parameters' estimation of the image from one representative sample.

Results of different multisource and multi-sensor images were presented in this work show the advantage of Bootstrap approach in both image fusion and even in image segmentation. Obtained results of our work show that using the BSEM approach in image fusion improves performances of estimated parameters which involve amelioration of the fused image quality, and reduces the computing time during the fusion process. Finally we noted that the obtained results of BSEM algorithm are better than SEM and WT approaches.

Further research will focus on how to integrate the probabilistic approaches (BEM, BSEM) with the MultiResolution (MR) methods such as Contourlet Transform (CT) and Nonsubsampled Contourlet Transform (NSCT); with the aim to enhance the fused product.

\section{REFERENCES}

[1] Simone G, Farina A, Morabito, Serpico S, Bruzzon L .image fusion techniques for remote sensing applications. Inf fusion, 2002; 3: 315 .

[2] Zhang Z, Blum RS. A categorization of multiscale decomposition based image fusion scheme with a performance study for a digital camera application. In: Proceedings of the IEEE 1999; 87: 8: pp.1315-26.

[3] Lui G, Jing ZL, Li JX, Sun SY,Li ZH, Leung H. Image fusion based on estimation theory. In: Proceedings of the third international conference on machine learning and cybernetics 2004; pp. 26-9.

[4] Nikolov SG, Lews JJ, O’Callaghan RG, Bull DR, Canagarajah CN. Hybrid fused displas: between pixel-and region-based image fusion. $7^{\text {th }}$ International conference on information fusion, Stokholm, Sweden 2004

[5] Ambois C. Introduction a la reconnaissance statistique des formes. Centre de Géostatique, Ecole des mines de Paris, mars 1997.

[6] Bin L, Jiaxiong P. Image fusion method based on short support symmetric non-separable wavelet. Int J Wavelets Multi-Resolut Inf Process 2004; 2: 87-98.

[7] Zhang Z, Blum RS. Region-based image fusion scheme for concealed weapon detection. In: Proceedings of the $31^{\text {st }}$ annual conference on information sciences and systems, march 1997

[8] Lewis JJ, O'callaghan RJ, Nikolov SG, Bull DR, Canagarajah CN. Region based image fusion using complex wavelets. $7^{\text {th }}$ interna- tional conference on information fusion, Stockholm, Sweden, 28 June 2004.

[9] Akerman A. pyramidal techniques for multisensor fusion. In: Proceedings of SPIE. Sensor Fusion V, 1992; Vol.1828: pp.124-31.

[10] Sharma RK. Probabilistic model-based multisensor image fusion. PHD dissertation, Oregon graduate institute, Portland, OR, 1999.

[11] Sharma RK, Leen TK, Pavel M. probabilistic image sensor fusion: advances in neural information processing systems, the MIT Press 1999; Vol.11.

[12] Yang J, Blum RS. A statistical signal processing approach to image fusion for concealed weapon detection. In: Proceedings of the IEEE international conference on image processing, Rochester, NY 2002; pp. 513-16.

[13] McLachlan GJ, Krishnan T. The EM Algorithm and Extensions. John Wiley and Son's Inc., New York 1997.

[14] Kehtarnavaz N, Nakamura E. Generalization of the EM algorithm for mixture density estimation. Pattern Recognit Lett 1998; 19, 133-40.

[15] Delleji T, Zribi M, Ben Hamida A. Application of bootstrap approach to unsupervised statistical segmentation of satellite images. $4^{\text {th }}$ Inteenational Multi-Conference on Systems, Signal \& Devices, SSd'2007, Hammamet Tunisia 2007.

[16] Zribi M. Non parametric and unsupervised Bayesia classification with Bootstrap sampling. Image Vision Comput 2004; 22: 1-8.

[17] Efron B, Tibshirani RJ. An introduction to the bootstrap. Monogr Stat Appl Probabil, 57, 1993.

[18] Banga C. L'approche Bootstrap en analyse des images : application à la restitution de la cinétique de la fuite en choriorétinopathie sereuse centrale. Thèse de doctorat, université de Rennes I 1995.

[19] Masson P, Pieczynski W. SEM algorithm and unsupervised statistical segmentation of satellite images. IEEE Trans. Geosci. Remote Sens 1993; Vol. 31, pp.618-33.

[20] Celeux G, Diebolt J. The SEM algorithm: a probabilistic teacher algorithm derived from the EM algorithm for the mixture problem. Comput Stat Q 1985; 2 (1), pp. 73-82.

[21] Efron B. Bootstrap methods: another look at the Jackknife. Ann. Stat 1979; 7 (1).

[22] Buvat I. Introduction à l'approche Bootstrap. U494 INSERM 2005.

[23] Zribi M, Ghorbel F. An unsupervised and nonparametric Bayesian classifier. Pattern Recognit Lett 2003; Vol. 24: pp. 97-112.

[24] Peng A, Pieczynski W. Adaptive mixture estimation and unsupervised local Bayesian image segmentation, Graph. Models Image Process 1995; 57: pp. 389-99.

[25] Caillol H, Pieczynski W, Hillion A.Estimation of fuzzy Gaussian mixture and unsupervised statistical image segmentation. IEEE Trans Image Process 1997; 6: 425-40.

[26] Dempester AP, Laird NM, Rubin DB. Maximum likelihood from incomplete data via the EM algorithm. J R Stat Soc 1977; 39: 1-38.

[27] Cvejic N, Loza A, Bull D, Canagarajah N. A Novel Metric for Performance Evaluation of Image Fusion Algorithms. Trans Eng Comput Technol 2005: 7.

[28] Redner RA, Walker HF. Mixture densities, maximum likelihood and the EM algorithm. SIAM Rev 1984; 26: pp. 195-239.

[29] Yang J, Blum RS. A region based image fusion method using the expectation-maximization algorithm. Information Sciences and Systems, $40^{\text {th }}$ annual conference on 2006; pp. 468-73.

[30] Hill P, Canagarajah N, Bull D. Image fusion using complex wavelets. In: Proceedings of the $13^{\text {th }}$ British machine vision conference, University of Cardiff 2-5 September 2002

[31] Song H, Yu S, Song L, Yang X. Fusion of multispectral and panchromatic satellite images based on contourlet transform and local average gradient. Opt Eng 2007; 46 (2).

[32] Piella G, Heijmans H. A new quality metric for image fusion. Image processing Proceedings 2003; Vol. 3, pp. 173-6.

[33] Toet A, Franken EM. Perceptual of different image fusion schmes Displays 2003; 24(1): 15-37.

[34] Wang Z, Bovik ZA. A universal image quality index. IEEE signal process lett 2002; 9: 81-4.

[35] German A, Jenkin MR, Lesperance Y. Entropy-Based Image Merging.The 2nd canadian conference on computer and robot vision (CRV'05) Toronto, Ontario, Canada 2005; pp. 81-86.

[36] Wang Z, Bovik AC, Hamid RS. Image quality assessment: from error visibility to structural similarity. IEEE Trans on Image Process 2004; 13(4): pp.600-12. 
(C) Delleji et al.; Licensee Bentham Open

This is an open access article licensed under the terms of the Creative Commons Attribution Non-Commercial License (http: //creativecommons.org/licenses/by$\mathrm{nc} / 3.0 /$ ) which permits unrestricted, non-commercial use, distribution and reproduction in any medium, provided the work is properly cited. 